\title{
An LMI Approach to Guaranteed Cost Control Design for Teleoperation Systems
}

\author{
Yuling Li and Yixin Yin \\ School of Automation and Electrical Engineering, University of Science and Technology Beijing, Beijing 100083, China \\ Correspondence should be addressed to Yuling Li; lyl8ustb@gmail.com
}

Received 18 May 2015; Revised 21 July 2015; Accepted 22 July 2015

Academic Editor: Mitsuhiro Okayasu

Copyright ( $) 2015$ Y. Li and Y. Yin. This is an open access article distributed under the Creative Commons Attribution License, which permits unrestricted use, distribution, and reproduction in any medium, provided the original work is properly cited.

\begin{abstract}
A procedure for the guaranteed cost control design of delayed bilateral teleoperation systems with nonlinear external forces is proposed. The assumption that the external forces are nonlinear functions of velocities and/or positions of local devices and that one part of these forces satisfies sector condition has been made. A stability criterion is formulated firstly and then the optimal guaranteed cost controller is obtained by solving the equivalent convex optimization problem in the form of linear matrix inequalities (LMIs). The controller preserves closed-loop stability regardless of the delay length. The behavior of the resulting teleoperation system is illustrated in simulations.
\end{abstract}

\section{Introduction}

Teleoperation systems enable humans to extend their capacity to manipulate remote interfaces with better safety, at less cost, and even with better accuracy. Their rich applications vary from nuclear operations and space and underwater exploration to medical surgery; see $[1,2]$ and references therein. In such an application, a human operator uses an interface referred to as a "master device" to conduct tasks by remotely controlling a "slave device" at the distant or hazardous environment. The general bilateral system setup is depicted in Figure 1. The main objectives of the control design for bilateral teleoperation systems are position coordination between the master and the slave and accurate haptic display of environment forces to the human operator. However, due to the very nature of teleoperation, time delays associated with communication between the local and the remote sites are inevitable. Handling the delays is especially problematic in the case of bilateral teleoperation, where the measurements are communicated in both directions to allow haptic feedback. The commonly proposed approaches to deal with bilateral teleoperation with time delays in the communication channel are mainly based on scattering theory formalism [3] or wave variable concept [4], which are called passivity-based control approaches. These controllers render the communication link passive and thus guarantee stable bilateral teleoperation. More recent research focused on improving tracking performance for constant and time varying delays and increasing the transparency of the teleoperator system and also the extensions to task space and network system control. For example, constant delays in the communication channel were considered in $[2,5]$, while [68] dealed with the communication channel with time varying time delays. Data loss and packet-switched network were considered in [9], where a passivity-based framework was proposed, and extension to the case where communication channel is a packet-switched network was also provided. Reference [10] used a modified Smith predictor, a kalman filter, and an energy regulator to control a teleoperator over Internet-based communication. Both quantization and time delay issues for teleoperation systems over networks were studied in [11]. Control design for bilateral teleoperation systems in task space was considered in $[12,13]$, where the task space position tracking was guaranteed. Another research related to task space control design of teleoperation systems can be found in $[14,15]$. In addition to the above mentioned results, passivity-based controllers relying on damping injection $[5,16]$, passive output synchronization $[16$, 17], and adaptive control [18] were also developed, recently. 


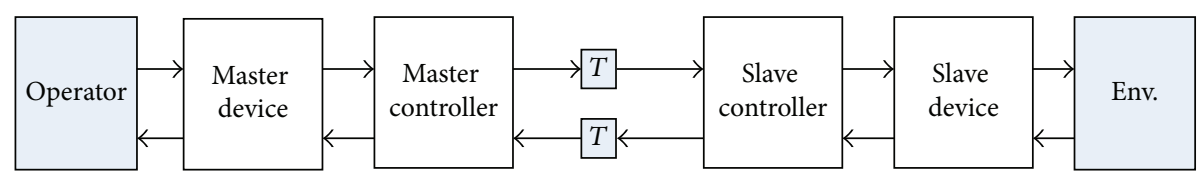

Figure 1: Bilateral teleoperation setup.

Several other approaches were proposed to deal with delays and are reviewed in [19].

Passivity-based controllers have the advantage of being able to guarantee stability independent of the user and environment dynamics; however, when controlling a real teleoperator, it is desirable to design a controller which not only guarantees the stability of the closed-loop system but also guarantees adequate level of performance. In this work a guaranteed cost control design is proposed for teleoperation systems with delays to guarantee a specific level of performance. We suppose that the external forces are nonlinear, while one part of them satisfies sector condition. Note that only one part of these forces satisfies a sector condition with respect to the output, which means that other parts may not be passive. Thus passivity is actually not required in our case. The position/velocity tracking performance is optimized by using the information of sector bounds. Constant and symmetric communication time delays are assumed to simplify the analysis; extensions for time varying delays are similar and easy to be realized.

The rest of the paper is organized as follows. In Section 2, the teleoperation model is generalized and then the problem formulation is given in Section 3. The controller design and its main results are given in Section 4, following which the simulation result is given to show the effectiveness of the proposed method in Section 5. Finally, the conclusion is included in Section 6.

The notation used throughout the paper is standard. $\mathbb{R}$ denotes the set of $n$-dimensional real vectors. For a real symmetric matrix $\mathbf{P}$, the notation of $\mathbf{P}>0(\mathbf{P}<0)$ is used to denote positive (negative) definiteness. I is used to denote the identity matrix of appropriate dimensions, $\mathbf{0}$ is the zero matrix of appropriate dimension, and $*$ is used to represent a block matrix which is readily referred to by symmetry. For a matrix $\mathbf{S}, \mathbf{S}^{T}$ denotes its transpose. For any function $\mathbf{f}:[0, \infty) \rightarrow \mathbb{R}^{n}$, the $\mathscr{L}_{\infty}$-norm is defined as $\|\mathbf{f}\|_{\infty}=\sup _{t \in[0, \infty)}|\mathbf{f}(t)|$ and the $\mathscr{L}_{2}$-norm is defined as $\|\mathbf{f}\|_{2}=$ $\left[\int_{0}^{\infty}|\mathbf{f}(t)|^{2} d t\right]^{1 / 2} \cdot \mathscr{L}_{2}$ and $\mathscr{L}_{\infty}$ spaces are defined as the sets $\left\{\mathbf{f}:[0, \infty) \rightarrow \mathbb{R}^{n}:\|\mathbf{f}\|_{2}<\infty\right\}$ and $\left\{\mathbf{f}:[0, \infty) \rightarrow \mathbb{R}^{n}:\right.$ $\left.\|\mathbf{f}\|_{\infty}<\infty\right\}$, respectively.

\section{Preliminaries: Bilateral System Modeling}

Consider a simple teleoperation system as follows:

$$
\begin{aligned}
\mathbf{m}_{m} \ddot{\mathbf{q}}_{m}+\mathbf{b}_{m} \dot{\mathbf{q}}_{m}+\mathbf{k}_{m} \mathbf{q}_{m} & =\mathbf{J}_{m}^{T}\left(\mathbf{q}_{m}\right) \mathbf{f}_{h}+\mathbf{u}_{m}, \\
\mathbf{m}_{s} \ddot{\mathbf{q}}_{s}+\mathbf{b}_{s} \dot{\mathbf{q}}_{s}+\mathbf{k}_{s} \mathbf{q}_{m} & =\mathbf{J}_{s}^{T}\left(\mathbf{q}_{s}\right) \mathbf{f}_{e}+\mathbf{u}_{s},
\end{aligned}
$$

where $\mathbf{q}_{i}, \dot{\mathbf{q}}_{i}, \ddot{\mathbf{q}}_{i} \in \mathbb{R}^{n}$ are the joint positions, velocities, and accelerations of the master and slave devices with $i=m$ or $s$ representing master or slave robot manipulators, respectively. Similarly, $\mathbf{m}_{i}, \mathbf{b}_{i}, \mathbf{k}_{i}$ are the effective mass, damping, and spring coefficients of the master and slave devices. External forces applied to the devices by the human operator and the environment are represented by $\mathbf{f}_{h}, \mathbf{f}_{e}$, respectively, while $\mathbf{J}_{m}\left(\mathbf{q}_{m}\right)$ and $\mathbf{J}_{s}\left(\mathbf{q}_{s}\right)$ are the Jacobian matrices of the master and the slave, respectively. $\mathbf{u}_{m}, \mathbf{u}_{s}$ stand for the control signals.

Defining $\boldsymbol{\xi}_{i}=\left[\boldsymbol{\xi}_{i 1}^{T}, \boldsymbol{\xi}_{i 2}^{T}\right]^{T}:=\left[\mathbf{q}_{i}^{T}, \dot{\mathbf{q}}_{i}^{T}\right]^{T}, \mathbf{y}_{i}=\mathbf{q}_{i}$ for each $i=m, s$, and $\mathbf{w}_{m}=\mathbf{J}_{m}^{T}\left(\mathbf{q}_{m}\right) \mathbf{f}_{h}, \mathbf{w}_{s}=\mathbf{J}_{s}^{T}\left(\mathbf{q}_{s}\right) \mathbf{f}_{e}$, a minimal statespace realization of system (1) is reformulated as

$$
\begin{aligned}
\dot{\boldsymbol{\xi}}_{i}(t) & =\mathbf{A}_{i} \boldsymbol{\xi}_{i}(t)+\mathbf{B}_{i w} \mathbf{w}_{i}(t)+\mathbf{B}_{i u} \mathbf{u}_{i}(t) \\
\mathbf{y}_{i} & =\mathbf{C}_{i y} \boldsymbol{\xi}_{i}(t)
\end{aligned}
$$

where

$$
\begin{aligned}
\mathbf{A}_{i} & =\left[\begin{array}{cc}
\mathbf{0} & \mathbf{I} \\
-\mathbf{m}_{i}^{-1} \mathbf{k}_{i} & -\mathbf{m}_{i}^{-1} \mathbf{b}_{i}
\end{array}\right], \\
\mathbf{B}_{i w} & =\left[\begin{array}{c}
\mathbf{0} \\
\mathbf{m}_{i}^{-1}
\end{array}\right], \\
\mathbf{B}_{i u} & =\left[\begin{array}{c}
\mathbf{0} \\
\mathbf{m}_{i}^{-1}
\end{array}\right], \\
\mathbf{C}_{i y} & =\left[\begin{array}{ll}
\mathbf{I} & \mathbf{0}
\end{array}\right] .
\end{aligned}
$$

\section{Problem Formulation}

The problem formulation based on the following assumptions is considered:

(A1) The forward/backward time delays in the communication channels are the same and are denoted by $\tau$.

(A2) The positions and velocities are available for measurement.

Remark 1. Assumptions A1 and A2 are very general in the control of teleoperation systems. Assumption A1 is for the simplification of the analysis, while the results in this paper can be easily extended to the case with asymmetric time varying delays.

The states and the input of the teleoperation system are defined as

$$
\begin{aligned}
& \mathbf{x}(t)=\left[\boldsymbol{\xi}_{m}^{T}(t), \boldsymbol{\xi}_{s}^{T}(t)\right]^{T}, \\
& \mathbf{u}(t)=\left[\mathbf{u}_{m}^{T}(t), \mathbf{u}_{s}^{T}(t)\right]^{T},
\end{aligned}
$$


and the output is defined as

$$
\mathbf{z}=\left[\mathbf{q}_{s}^{T}(t-\tau)-\mathbf{q}_{m}^{T}(t-\tau), \dot{\mathbf{q}}_{s}^{T}(t-\tau)-\dot{\mathbf{q}}_{m}^{T}(t-\tau)\right]^{T},
$$

and the evolution of the states is governed by

$$
\begin{aligned}
\dot{\mathbf{x}}(t) & =\mathbf{A} \mathbf{x}(t)+\mathbf{A}_{1} \mathbf{x}(t-\tau)+\mathbf{B} \mathbf{u}(t)+\mathbf{D W}(t), \\
\mathbf{z}(t) & =\mathbf{H}_{d} \mathbf{x}(t-\tau), \\
\mathbf{x}(t) & =\mathbf{x}_{0}(t), \quad \forall t \in[-\tau, 0],
\end{aligned}
$$

with $\mathbf{W}(t)=\left[\mathbf{w}_{m}^{T}(t), \mathbf{w}_{s}^{T}(t)\right]^{T}$, where

$$
\begin{aligned}
& \mathbf{A}=\left[\begin{array}{cc}
\mathbf{A}_{m} & \mathbf{0} \\
\mathbf{0} & \mathbf{A}_{s}
\end{array}\right], \\
& \mathbf{B}=\left[\begin{array}{cc}
\mathbf{B}_{m u} & \mathbf{0} \\
\mathbf{0} & \mathbf{B}_{s u}
\end{array}\right] \text {, } \\
& \mathbf{A}_{1}=\mathbf{0} \text {, } \\
& \mathbf{D}=\left[\begin{array}{cc}
\mathbf{B}_{m w} & \mathbf{0} \\
\mathbf{0} & \mathbf{B}_{s w}
\end{array}\right], \\
& \mathbf{H}_{d}=\left[\begin{array}{cccc}
-\mathbf{I} & \mathbf{0} & \mathbf{I} & \mathbf{0} \\
\mathbf{0} & -\mathbf{I} & \mathbf{0} & \mathbf{I}
\end{array}\right] .
\end{aligned}
$$

Note that $\mathbf{A}_{1}=\mathbf{0}$ in dynamics (6) is included here for the convenience of stability analysis, and the details will be found in the following sections.

The following assumption is furthermore given:

(A3) $\mathbf{W}(t)$ can be modeled as

$$
\mathbf{W}(t)=\mathbf{F}^{\star}(t)+\mathbf{F}(t, \mathbf{z}(t)),
$$

where $\mathbf{F}^{\star}(t)$ is continuous uniformly bounded function satisfying $\int_{0}^{\infty}\left\|\mathbf{F}^{\star}(s)\right\|_{2}^{2} d s \leqslant \Lambda$ and $\mathbf{F}(t, \mathbf{z}(t))$ is a nonlinear function of $\mathbf{z}(t)$ and it is piecewise continuous in $t$, globally Lipschitz in $\mathbf{z}(t)$ with $\mathbf{F}(t, 0)=0$, while satisfying the following sector condition (a) or (b) for $\forall t \geqslant 0$. Consider

(a)

$$
\mathbf{F}^{T}(t, \mathbf{z}(t))[\mathbf{F}(t, \mathbf{z}(t))-\mathbf{K z}(t)] \leqslant 0,
$$

(b)

$$
\left(\mathbf{F}(t, \mathbf{z}(t))-\mathbf{K}_{1} \mathbf{z}(t)\right)^{T}\left(\mathbf{F}(t, \mathbf{z}(t))-\mathbf{K}_{2} \mathbf{z}(t)\right) \leqslant 0,
$$

where $\mathbf{K}, \mathbf{K}_{1}, \mathbf{K}_{2}$ are constant real matrices of appropriate dimensions.

Remark 2. Equations (11) and (12) are special cases of the prevailing passivity-type assumption. The sector bounds $\mathbf{K}_{1}$ and $\mathbf{K}_{2}$ are introduced to provide bounding information of the nonlinearities in the external forces, which also make the following optimization steps possible. Note that external forces $\mathbf{W}(t)$ do not satisfy passivity assumption due to the time varying $\mathbf{F}^{\star}(t)$. However, when $\mathbf{F}^{\star}(t) \equiv 0$, Assumption A3 reduces to the prevailing passivity-type assumption.
The state feedback controllers for the teleoperation system (1) or (6)-(8) are proposed as follows:

$$
\begin{aligned}
\mathbf{u}_{m}= & \mathbf{L}_{11}\left[\mathbf{q}_{m}^{T}(t-\tau), \dot{\mathbf{q}}_{m}^{T}(t-\tau)\right]^{T} \\
& +\mathbf{L}_{12}\left[\mathbf{q}_{s}^{T}(t-\tau), \dot{\mathbf{q}}_{s}^{T}(t-\tau)\right]^{T}, \\
\mathbf{u}_{s}= & \mathbf{L}_{21}\left[\mathbf{q}_{m}^{T}(t-\tau), \dot{\mathbf{q}}_{m}^{T}(t-\tau)\right]^{T} \\
& +\mathbf{L}_{22}\left[\mathbf{q}_{s}^{T}(t-\tau), \dot{\mathbf{q}}_{s}^{T}(t-\tau)\right]^{T},
\end{aligned}
$$

where $\mathbf{L}_{11}, \mathbf{L}_{12}, \mathbf{L}_{21}, \mathbf{L}_{22}$ are matrices to be designed. Hence the control law can be rewritten as

$$
\mathbf{u}(t)=\mathbf{L x}(t-\tau),
$$

where $\mathbf{L}=\left[\begin{array}{ll}\mathbf{L}_{11} & \mathbf{L}_{12} \\ \mathbf{L}_{21} & \mathbf{L}_{22}\end{array}\right]$.

Remark 3. Note that pretended time delays are intentionally introduced in system output (5) and controllers (13) and (14); by doing this, it avoids the case that the resulting setup follows into a group of controller design problems with intrinsically quadratically invariant constraints on the controller parameters; see [20] for more discussions. The basic idea is to design the guaranteed cost controller for system (6)-(8) under the worst case; that is, there are delays both in the local and in the remote variables; then better performance can be achieved when local variables actually have no time delays. For a practical teleoperation system of which local state variables do not have time delays, the stability and performance will be guaranteed as well. In the simulation, the time delays at the local sides were removed to show the stability.

To introduce the main objective of this paper, a cost function associated with system (6)-(8) is introduced:

$$
J=\int_{0}^{\infty}\left[\mathbf{z}^{T}(\theta) \mathbf{Q z}(\theta)+\mathbf{u}^{T}(\theta) \mathbf{R u}(\theta)\right] d \theta,
$$

where $\mathbf{Q}, \mathbf{R}$ are given positive-definite symmetric matrices.

Hence, the main objective of this paper is to design a guaranteed cost control law (14) for the teleoperation system (1) with the human force and environmental force satisfying Assumption A3 such that the closed-loop system is stable with an associated cost $J$ less than a guaranteed cost $J^{\star}$. The problem setup is shown in Figure 2.

\section{Controller Design}

The case when the nonlinear function $\mathbf{F}(t, \mathbf{z}(t))$ satisfies (11) is firstly considered and the following result is obtained. After this, the result will be extended to the general case in which (12) is satisfied.

Theorem 4. For the teleoperation system (6)-(8) with external forces satisfying Assumption A3 with the simple sector condition (11), the guaranteed cost controller (14) exists if there 


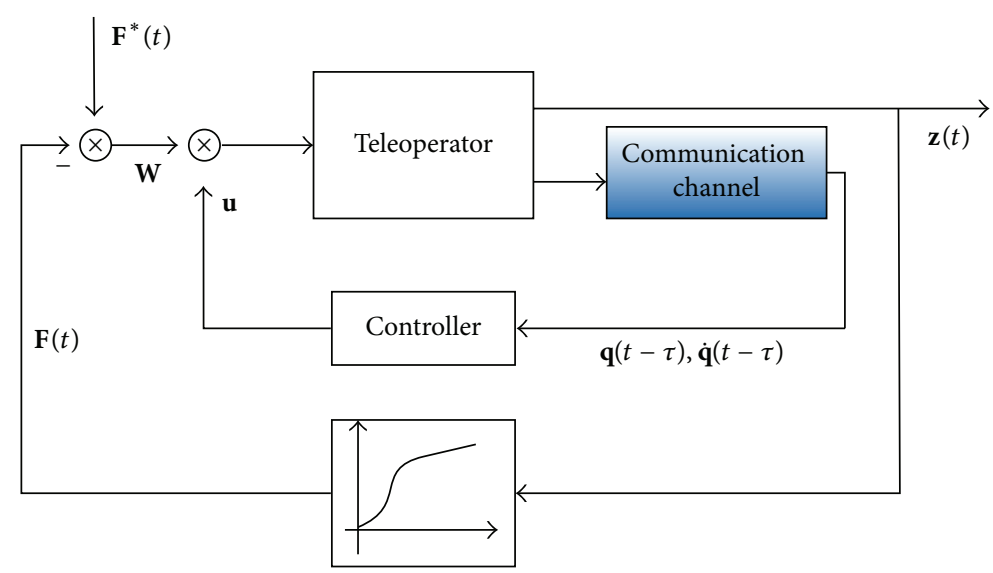

Figure 2: Problem formulation.

exist symmetric positive-definite matrices $\mathbf{P}, \mathbf{S} \in \mathbb{R}^{4 n \times 4 n}$ and scalars $\varepsilon>0, \rho>0$ such that

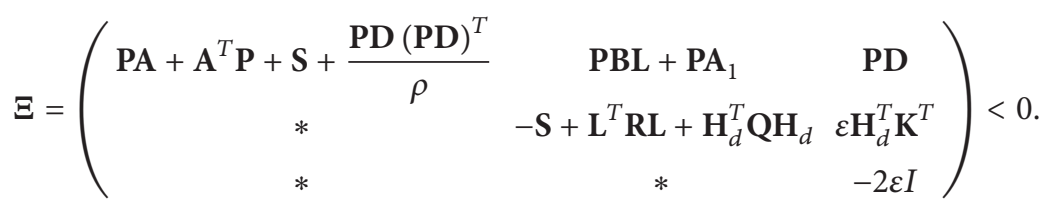

Proof. Choose a Lyapunov-Krasovskii functional candidate as

$$
V(t)=\mathbf{x}^{T}(t) \mathbf{P} \mathbf{x}(t)+\int_{t-\tau}^{t} \mathbf{x}^{T}(\theta) \mathbf{S} \mathbf{x}(\theta) d \theta,
$$

where $\mathbf{P}>0, \mathbf{S}>0$. Taking derivative of $V(t)$ with respect to $t$ along the trajectory of system (6)-(8) yields

$$
\begin{aligned}
\dot{V}(t)= & \dot{\mathbf{x}}^{T}(t) \mathbf{P} \mathbf{x}(t)+\mathbf{x}^{T}(t) \mathbf{P} \dot{\mathbf{x}}(t)+\mathbf{x}^{T}(t) \mathbf{S} \mathbf{x}(t) \\
& -\mathbf{x}^{T}(t-\tau) \mathbf{S} \mathbf{x}(t-\tau) \\
= & \mathbf{x}^{T}(t)\left(\mathbf{P A}+\mathbf{A}^{T} \mathbf{P}+\mathbf{S}\right) \mathbf{x}(t) \\
& +2 \mathbf{x}^{T}(t)\left(\mathbf{P} \mathbf{A}_{1}+\mathbf{P B L}\right) \mathbf{x}(t-\tau) \\
& -\mathbf{x}^{T}(t-\tau) \mathbf{S} \mathbf{x}(t-\tau)+2 \mathbf{x}^{T}(t) \mathbf{P D F}^{\star}(t) \\
& +2 \mathbf{x}^{T}(t) \mathbf{P D F}(t, \mathbf{z}(t)) .
\end{aligned}
$$

Denote $\boldsymbol{\eta}(t)=\left[\mathbf{x}^{T}(t), \mathbf{x}^{T}(t-\tau), \mathbf{F}^{T}(t, \mathbf{z}(t))\right]^{T}$, and one has $\dot{V}(t)$

$$
\begin{aligned}
= & \boldsymbol{\eta}^{T}(t)\left[\begin{array}{ccc}
\mathbf{A}^{T} \mathbf{P}+\mathbf{P A}+\mathbf{S} & \mathbf{P A}_{1}+\mathbf{P B L} & \mathbf{P D} \\
* & -\mathbf{S} & \mathbf{0} \\
* & * & \mathbf{0}
\end{array}\right] \boldsymbol{\eta}(t) \\
& +2 \mathbf{x}^{T}(t) \mathbf{P D F}^{\star}(t) .
\end{aligned}
$$

From (11), one has

$$
\mathbf{F}^{T}(t, \mathbf{z}(t))\left(\mathbf{F}(t, \mathbf{z}(t))-\mathbf{K H}_{d} \mathbf{x}(t-\tau)\right) \leqslant 0 .
$$

Employ the inequality obtained by completion of squares:

$$
\begin{aligned}
2 \mathbf{x}^{T}(t) \mathbf{P D F}^{\star}(t) \leqslant & \frac{\mathbf{x}^{T}(t)(\mathbf{P D})(\mathbf{P D})^{T} \mathbf{x}(t)}{\rho} \\
& +\rho \mathbf{F}^{\star}(t)^{T} \mathbf{F}^{\star}(t),
\end{aligned}
$$

where $\rho>0$, and then from (19), (20), and (21) one obtains that

$$
\begin{aligned}
\dot{V}(t) \leqslant & \boldsymbol{\eta}^{T}(t) \Xi \boldsymbol{\eta}(t)-\mathbf{z}^{T}(t) \mathbf{Q} \mathbf{z}(t)-\mathbf{u}^{T}(t) \mathbf{R} \mathbf{u}(t) \\
& +2 \varepsilon \mathbf{F}^{T}(t, \mathbf{z}(t)) \mathbf{F}(t, \mathbf{z}(t)) \\
& -2 \varepsilon \mathbf{F}^{T}(t, \mathbf{z}(t)) \mathbf{K} \mathbf{H}_{d} \mathbf{x}(t-\tau) \\
& +\rho \mathbf{F}^{\star}(t)^{T} \mathbf{F}^{\star}(t)
\end{aligned}
$$

hence (11) implies that

$$
\begin{aligned}
\dot{V}(t) \leqslant & \boldsymbol{\eta}^{T}(t) \Xi \boldsymbol{\eta}(t)-\mathbf{z}^{T}(t) \mathbf{Q z}(t)-\mathbf{u}^{T}(t) \mathbf{R u}(t) \\
& +\rho \mathbf{F}^{\star}(t)^{T} \mathbf{F}^{\star}(t) .
\end{aligned}
$$


By integrating both sides of inequality (23) from 0 to $t$, one has

$$
\begin{aligned}
V(t) & -V(0) \\
\leqslant & \lambda_{\max }\{\boldsymbol{\Xi}\} \int_{0}^{t} \boldsymbol{\eta}^{T}(s) \boldsymbol{\eta}(s) d s \\
& -\int_{0}^{t}\left(\mathbf{z}^{T}(s) \mathbf{Q z}(s)+\mathbf{u}^{T}(s) \mathbf{R u}(s)\right) d s \\
& +\int_{0}^{t} \mathbf{F}^{\star}(s)^{T} \mathbf{F}^{\star}(s) d s \\
< & \lambda_{\max }\{\boldsymbol{\Xi}\}\|\boldsymbol{\eta}\|_{2}^{2} \\
& -\int_{0}^{t}\left(\mathbf{z}^{T}(s) \mathbf{Q z}(s)+\mathbf{u}^{T}(s) \mathbf{R u}(s)\right) d s+\rho \Lambda \\
< & \lambda_{\max }\{\boldsymbol{\Xi}\}\|\boldsymbol{\eta}\|_{2}^{2}+\rho \Lambda .
\end{aligned}
$$

Now, in view of (16) and (24), one can conclude that $\dot{V}(t)$ is negative if $\|\boldsymbol{\eta}\|_{2}>\sqrt{2 \rho \Lambda /-\lambda_{\max }\{\boldsymbol{\Xi}\}}$, and thus this implies that $V(t) \in \mathscr{L}_{\infty}, \boldsymbol{\eta}(t) \in \mathscr{L}_{2}$, and hence $\mathbf{x}(t) \in \mathscr{L}_{2} \cap \mathscr{L}_{\infty}$, $\mathbf{x}(t-\tau) \in \mathscr{L}_{2} \cap \mathscr{L}_{\infty}$. Furthermore, one could easily get that

$$
\begin{aligned}
J & \leqslant V(0)+\rho \Lambda \\
& =\mathbf{x}^{T}(0) \mathbf{P} \mathbf{x}(0)+\int_{-\tau}^{0} \mathbf{x}^{T}(\theta) \mathbf{S} \mathbf{x}(\theta) d \theta+\rho \Lambda .
\end{aligned}
$$

This completes the proof.

Based on the stability criterion above, the original problem of designing a guaranteed cost controller (14) can be transformed to a convex optimization problem, which is presented below.

Theorem 5. For the teleoperation system (6)-(8) with external forces satisfying Assumption A3 with the simple sector condition (11), the control law (14) is an optimal memoryless guaranteed cost control law which ensures the minimization of the guaranteed cost (15) if the following convex optimization problem,

$$
\min _{\chi_{i}(i=1,2,3,4), \alpha, \mathbf{M}} \alpha+\operatorname{tr}(\mathbf{M})
$$

subject to

(1)

$$
\left[\begin{array}{ccccccc}
\mathbf{A} \chi_{1}+\chi_{1} \mathbf{A}^{T} & \mathbf{A}_{1} \chi_{2}+\mathbf{B} \chi_{4} & \chi_{3} \mathbf{D} & \mathbf{0} & \chi_{1} & \mathbf{0} & \mathbf{D} \\
* & -\chi_{2} & \chi_{2} \mathbf{H}_{d}^{T} \mathbf{K}^{T} & \chi_{4}^{T} & 0 & \chi_{2} \mathbf{H}_{d}^{T} & 0 \\
* & * & -2 \chi_{3} \mathbf{I} & \mathbf{0} & \mathbf{0} & \mathbf{0} & \mathbf{0} \\
* & * & * & -\mathbf{R}^{-1} & \mathbf{0} & \mathbf{0} & \mathbf{0} \\
* & * & * & * & -\chi_{2} & \mathbf{0} & \mathbf{0} \\
* & * & * & * & * & -\mathbf{Q}^{-1} & \mathbf{0} \\
* & * & * & * & * & * & -\rho \mathbf{I}
\end{array}\right]<0,
$$

(2) the matrices $\chi_{1}, \chi_{2}$ which are symmetric, and

$$
\begin{gathered}
\chi_{1}>0, \\
\chi_{2}>0, \\
\chi_{3}>0, \\
\rho>0,
\end{gathered}
$$

(3)

(4)

$$
\left[\begin{array}{cc}
\alpha & \mathbf{x}_{0}^{T}(0) \\
\mathbf{x}_{0}(0) & \chi_{1}
\end{array}\right]>0,
$$

$$
\left[\begin{array}{ll}
\mathbf{M} & \mathbf{N}^{T} \\
\mathbf{N} & \chi_{2}
\end{array}\right]>0
$$

has a solution $\chi_{1}, \chi_{2}, \chi_{3}, \chi_{4}, \rho$. Here, $\operatorname{tr}(\cdot)$ denotes the trace of the matrix $\cdot$ and $\int_{-\tau}^{0} \mathbf{x}_{0}(\theta) \mathbf{x}_{0}^{T}(\theta)=\mathbf{N N}^{T}$. In addition, the controller gains in (14) are given as $\mathbf{L}=\chi_{4} \chi_{2}^{-1}$.

Proof. It follows from the Schur complement that (16) is equivalent to

$$
\left[\begin{array}{ccccccc}
\mathbf{P A}+\mathbf{A}^{T} \mathbf{P} & \mathbf{P A}_{1}+\mathbf{P B L} & \mathbf{P D} & \mathbf{0} & \mathbf{I} & \mathbf{0} & \mathbf{P D} \\
* & -\mathbf{S} & \varepsilon \mathbf{H}_{d}^{T} \mathbf{K}^{T} & \mathbf{L}^{T} & \mathbf{0} & \mathbf{H}_{d}^{T} & \mathbf{0} \\
* & * & -2 \varepsilon \mathbf{I} & \mathbf{0} & \mathbf{0} & \mathbf{0} & \mathbf{0} \\
* & * & * & -\mathbf{R}^{-1} & \mathbf{0} & \mathbf{0} & \mathbf{0} \\
* & * & * & * & -\mathbf{S}^{-1} & \mathbf{0} & \mathbf{0} \\
* & * & * & * & * & -\mathbf{Q}^{-1} & \mathbf{0} \\
* & * & * & * & * & * & -\rho \mathbf{I}
\end{array}\right]
$$


Pre- and postmultiplying both sides of (31) by

$$
\left[\begin{array}{ccccccc}
\mathbf{P}^{-1} & \mathbf{0} & \mathbf{0} & \mathbf{0} & \mathbf{0} & \mathbf{0} & \mathbf{0} \\
* & \mathbf{S}^{-1} & \mathbf{0} & \mathbf{0} & \mathbf{0} & \mathbf{0} & \mathbf{0} \\
* & * & \varepsilon^{-1} \mathbf{I} & \mathbf{0} & \mathbf{0} & \mathbf{0} & \mathbf{0} \\
* & * & * & \mathbf{I} & \mathbf{0} & \mathbf{0} & \mathbf{0} \\
* & * & * & * & \mathbf{I} & \mathbf{0} & \mathbf{0} \\
* & * & * & * & * & \mathbf{I} & \mathbf{0} \\
* & * & * & * & * & * & \mathbf{I}
\end{array}\right]
$$

yield LMI (27), where $\chi_{1}=\mathbf{P}^{-1}, \chi_{2}=\mathbf{S}^{-1}, \chi_{3}=1 / \varepsilon, \chi_{4}=$ $\mathbf{L S}^{-1}$. By applying Theorem 3 in [21], convex optimization problem (26) was obtained. It completes the proof.

For the nonlinearity $\mathbf{F}(t, \mathbf{z}(t))$ satisfying the more general sector condition (12), by applying the idea known as loop transformation [22], one can conclude that the absolute stability of system (6)-(8) with the nonlinearity within the sector $\left[\mathbf{K}_{1}, \mathbf{K}_{2}\right]$ is equivalent to that of the following system:

$$
\begin{aligned}
\dot{\mathbf{x}} & =\mathbf{A} \mathbf{x}+\mathbf{D K}_{1} \mathbf{H}_{d} \mathbf{x}(t-\tau)+\mathbf{B u}+\mathbf{D} \widetilde{\mathbf{W}}, \\
\mathbf{z} & =\mathbf{H}_{d} \mathbf{x}(t-\tau), \\
\widetilde{\mathbf{W}} & =\widetilde{\mathbf{F}}(t, \mathbf{z}(t))+\mathbf{F}^{\star}(t), \\
\mathbf{x}(t) & =\mathbf{x}_{0}(t), \quad \forall t \in[-\tau, 0],
\end{aligned}
$$

where $\widetilde{\mathbf{F}}(t, \mathbf{z}(t))=\mathbf{F}(t, \mathbf{z}(t))-\mathbf{K}_{1} \mathbf{z}(t)$ satisfies the sector condition

$$
\widetilde{\mathbf{F}}^{T}(t, \mathbf{z}(t))\left[\widetilde{\mathbf{F}}(t, \mathbf{z}(t))-\left(\mathbf{K}_{2}-\mathbf{K}_{1}\right) \mathbf{z}(t)\right] \leqslant 0
$$

for $\forall t \geqslant 0$. Hence by Theorem 4 the following result is obtained.

Proposition 6. For the teleoperation system (6)-(8) with external forces satisfying Assumption A3 with the sector condition (12), the guaranteed cost controller (14) exists if there exist symmetric $\chi_{1}>0, \chi_{2}>0, \chi_{3}>0$ such that

$$
\left[\begin{array}{ccccccc}
\boldsymbol{\Omega}_{11} & \boldsymbol{\Omega}_{12} & \boldsymbol{\Omega}_{13} & \mathbf{0} & \chi_{1} & \mathbf{0} & \mathbf{D} \\
* & -\chi_{2} & \boldsymbol{\Omega}_{23} & \chi_{4}^{T} & \mathbf{0} & \chi_{2} \mathbf{H}_{d}^{T} & \mathbf{0} \\
* & * & -2 \chi_{3} \mathbf{I} & \mathbf{0} & \mathbf{0} & \mathbf{0} & \mathbf{0} \\
* & * & * & -\mathbf{R}^{-1} & \mathbf{0} & \mathbf{0} & \mathbf{0} \\
* & * & * & * & -\chi_{2} & \mathbf{0} & \mathbf{0} \\
* & * & * & * & * & -\mathbf{Q}^{-1} & \mathbf{0} \\
* & * & * & * & * & * & -\rho \mathbf{I}
\end{array}\right]<0,
$$

where

$$
\begin{aligned}
& \boldsymbol{\Omega}_{11}=\mathbf{A} \boldsymbol{\chi}_{1}+\chi_{1} \mathbf{A}^{T}, \\
& \boldsymbol{\Omega}_{12}=\mathbf{D} \mathbf{K}_{1} \mathbf{H}_{d} \boldsymbol{\chi}_{2}+\mathbf{B} \boldsymbol{\chi}_{4}, \\
& \boldsymbol{\Omega}_{13}=\chi_{3} \mathbf{D}, \\
& \boldsymbol{\Omega}_{23}=\chi_{2} \mathbf{H}_{d}^{T}\left(\mathbf{K}_{2}-\mathbf{K}_{1}\right)^{T} .
\end{aligned}
$$

Now, following Theorem 5, the guaranteed cost controller (14) for system (6)-(8) with nonlinearities (12) can be obtained by solving the optimization problem (26) with constraints (28), (29), (30), and (35).

Remark 7. The main results in this paper provide a delayindependent stability criterion for the nonlinear system (6)(8). If the Lyapunov-Krasovskii functional is chosen as $\widetilde{V}=$ $V+\int_{t-\tau}^{t}(\tau-t+\theta) \dot{\mathbf{x}}^{T}(\theta)\left(\tau \mathbf{S}_{1}\right) \dot{\mathbf{x}}(\theta) d \theta$, where $\mathbf{S}_{1}>0$ and $V$ is defined in (17), a delay-dependent stability criterion for the system can be obtained.

\section{Simulations and Results}

Consider a master-slave teleoperation system (1) with the effective mass $m_{m}=m_{s}=3 \mathrm{Kg}$, the damping $b_{m}=b_{s}=$ $80 \mathrm{Ns} / \mathrm{m}$, and the spring coefficients $k_{m}=k_{s}=45 \mathrm{~N} / \mathrm{m}$.

Hence it can be described by (6)-(8) with

$$
\mathbf{A}=\left[\begin{array}{cccc}
0 & 1.0000 & 0 & 0 \\
-15.0000 & -26.6667 & 0 & 0 \\
0 & 0 & 0 & 1.0000 \\
0 & 0 & -15.0000 & -26.6667
\end{array}\right]
$$

$\mathbf{A}_{1}=0$,

$$
\mathbf{B}=\left[\begin{array}{cc}
0 & 0 \\
0.3333 & 0 \\
0 & 0 \\
0 & 0.3333
\end{array}\right]
$$

$$
\mathbf{D}=\left[\begin{array}{cc}
0 & 0 \\
0.3333 & 0 \\
0 & 0 \\
0 & 0.3333
\end{array}\right]
$$

$$
\mathbf{H}_{d}=\left[\begin{array}{cccc}
-1 & 0 & 1 & 0 \\
1 & 0 & -1 & 0
\end{array}\right] \text {, }
$$

and the initial states are chosen as

$$
\begin{aligned}
& q_{m}(t)=e^{t+0.2}, \\
& q_{s}(t)=e^{2(t+0.2)}, \\
& \dot{q}_{m}(t)=e^{t+0.2}, \\
& \dot{q}_{s}(t)=2 e^{2(t+0.2),}
\end{aligned}
$$

$\forall t \in[-0.2,0]$ with the time delay $\tau=0.2 \mathrm{~s}$. Here the master and the slave with different initial positions and velocities are chosen to see the tracking performance. 


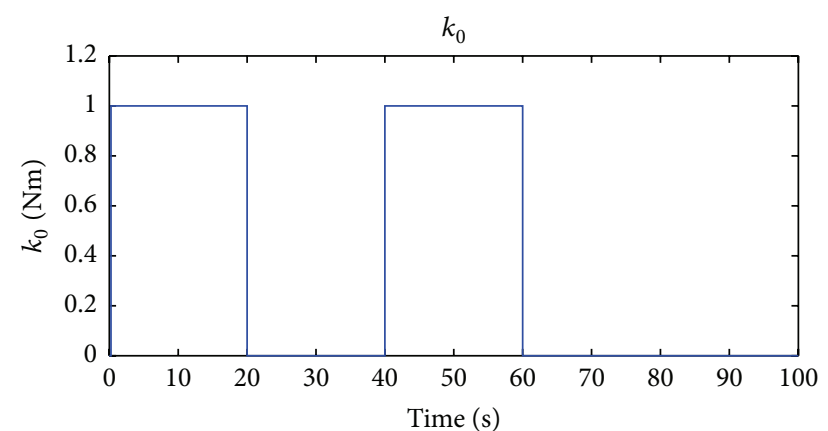

Figure 3: External force $k_{0}(t)$.

To access the teleoperation system behavior, the human force $w_{m}$ and the environment force $w_{s}$ are firstly chosen to be linear; that is,

$$
\begin{aligned}
w_{m}(t)= & a_{m} k_{0}(t)+k_{h_{p}}\left(q_{s}(t-\tau)-q_{m}(t-\tau)\right) \\
& +k_{h_{v}}\left(\dot{q}_{s}(t-\tau)-\dot{q}_{m}(t-\tau)\right), \\
w_{s}(t)= & a_{s} k_{0}(t)+k_{e_{p}}\left(q_{s}(t-\tau)-q_{m}(t-\tau)\right) \\
& +k_{e_{v}}\left(\dot{q}_{s}(t-\tau)-\dot{q}_{m}(t-\tau)\right),
\end{aligned}
$$

with $k_{0}(t)$ as the external input which is chosen to be the rectangle signal depicted in Figure 3, and $a_{m}=10, a_{s}=5$. The coefficients are chosen as $k_{h_{p}}=10, k_{e_{p}}=50, k_{h_{v}}=5$, and $k_{e_{v}}=80$. Hence, the nonlinearity satisfies (11) with

$$
\mathbf{K}=\left[\begin{array}{cc}
10 & 5 \\
50 & 80
\end{array}\right] \text {. }
$$

The cost function (15) is chosen with

$$
\begin{aligned}
& \mathbf{Q}=\left[\begin{array}{cc}
100 & 0 \\
0 & 1
\end{array}\right], \\
& \mathbf{R}=\left[\begin{array}{cc}
0.01 & 0 \\
0 & 0.01
\end{array}\right] .
\end{aligned}
$$

By Theorem 5 and solving the corresponding optimization problem by using YALMIP toolbox [23], the optimal guaranteed cost control law with the solution

$$
\mathbf{L}=\left[\begin{array}{cccc}
-9.9896 & -18.7494 & 9.9896 & 18.7494 \\
10.0104 & 18.7506 & -10.0104 & -18.7506
\end{array}\right]
$$

is obtained.

Noting the structure of the control gains in (42), one can rewrite (14) with parameter (42) as

$$
\begin{aligned}
u_{m}(t)= & 9.9896\left(q_{s}(t-\tau)-q_{m}(t)\right) \\
& +18.7494\left(\dot{q}_{s}(t-\tau)-\dot{q}_{m}(t)\right), \\
u_{s}(t)= & 10.0104\left(q_{m}(t-\tau)-q_{s}(t)\right) \\
& +18.7506\left(\dot{q}_{m}(t-\tau)-\dot{q}_{s}(t)\right) .
\end{aligned}
$$

It can be seen that the optimal controllers are ProportionalDerivative (PD) controllers. Note that the time delays in the local variables are omitted since in the simulations there is no time delay in the local variables. Applying controller (43) to the described system, the simulation results shown in Figures 4(a) and 4(b) are obtained. It can be seen that the master and the slave response stably. The positions and the velocities of the slave follow the master's motion. Specifically, the master and the slave move forward and backward with the increase and decrease of the human force, respectively. Let $\mathscr{f}(t)=\int_{0}^{t}\left[\mathbf{z}^{T}(\theta) \mathbf{Q z}(\theta)+\mathbf{u}^{T}(\theta) \mathbf{R u}(\theta)\right] d \theta$, the value of the cost $\mathscr{J}(t)$ can also be tracked in Figure 4(c), and the best cost is $\lim _{t \rightarrow+\infty} \mathscr{f}(t)=J^{*}=40$.

Now nonlinearities into the external forces are introduced; that is,

$$
\begin{gathered}
w_{m}(t)=a_{m} k_{0}(t)-k_{h} \tan ^{-1}\left(q_{s}(t-\tau)-q_{m}(t-\tau)\right), \\
w_{s}(t)=a_{s} k_{0}(t)+k_{e} \tan ^{-1}\left(q_{s}(t-\tau)-q_{m}(t-\tau)\right),
\end{gathered}
$$

where $\tan ^{-1}(\cdot)$ is the inverse function of $(\tan (\cdot))$ and $k_{h}=10$, $k_{e}=50$.

By Theorem 5 the optimal guaranteed cost control law with the solution

$$
\mathbf{L}=\left[\begin{array}{cccc}
-9.9894 & 0.0000 & 9.9894 & 0.0000 \\
10.0107 & 0.0000 & -10.0107 & 0.0000
\end{array}\right]
$$

is obtained and thus the equivalent controllers in the traditional form are

$$
\begin{aligned}
& u_{m}(t)=9.9894\left(q_{s}(t-\tau)-q_{m}(t)\right), \\
& u_{s}(t)=10.0107\left(q_{m}(t-\tau)-q_{s}(t)\right) .
\end{aligned}
$$

It can be seen that the optimal controllers are Proportional (P) controllers. Simulation results for the system with nonlinear external forces (44) are presented in Figures 5(a) and 5(b). There is still no time delay existing in the local variables, which is more realistic. It can be seen that the simulation results for the case when the external forces are nonlinear are similar to the results for the case when the external results are linear. This implies that the controller is valid for the system with nonlinear external forces. The value of the cost is also tracked in Figure 5(c), and the best cost is $J^{*}=4.3562$.

\section{Conclusion}

In this paper, a guaranteed cost control design procedure for delayed bilateral teleoperation systems is proposed. The assumption that there exist time delays in the local devices has been made to obtain the desired controllers under the worst case, and thus the quadratically invariant problem setup is avoided. Furthermore, only one part of the external forces is assumed to satisfy some section conditions, and thus the guaranteed cost controller is introduced. To obtain the guaranteed cost control gains, we firstly formulate a stability criterion and then convert it to a convex optimization problem which can be solved by linear matrix inequality (LMI) technique. Simulations for a simple example show that 


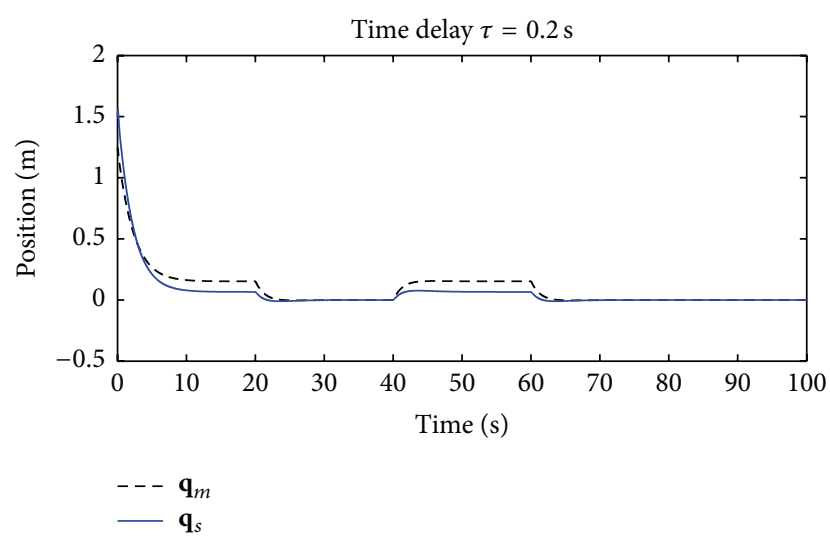

(a) Position tracking performance

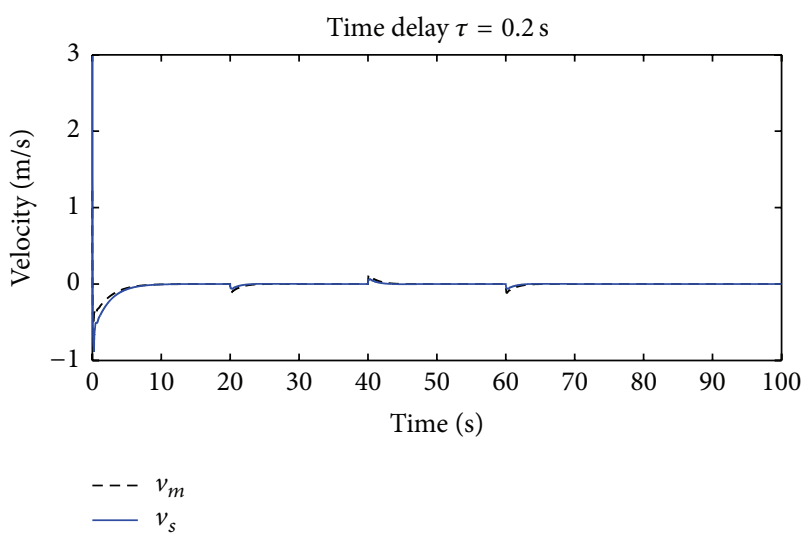

(b) Velocity tracking performance

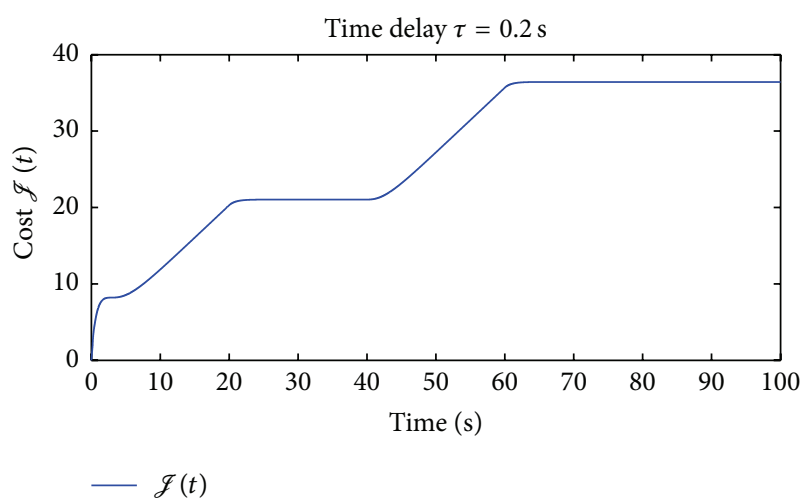

(c) Cost function $\mathscr{f}(t)$

FIGURE 4: Simulation results when the human and environment forces are linear.

the stability is guaranteed with very good performance when the designed controller is applied to the systems which do not actually have delays in the local devices. It is also found that the actual controllers obtained from our method are PD controllers or P controllers, but there is no need to pick any PD or P parameters in our method while guaranteed level of performance is satisfied. It is very beneficial for the control of teleoperation systems with high degrees of freedom in practical applications. Further study will focus on the

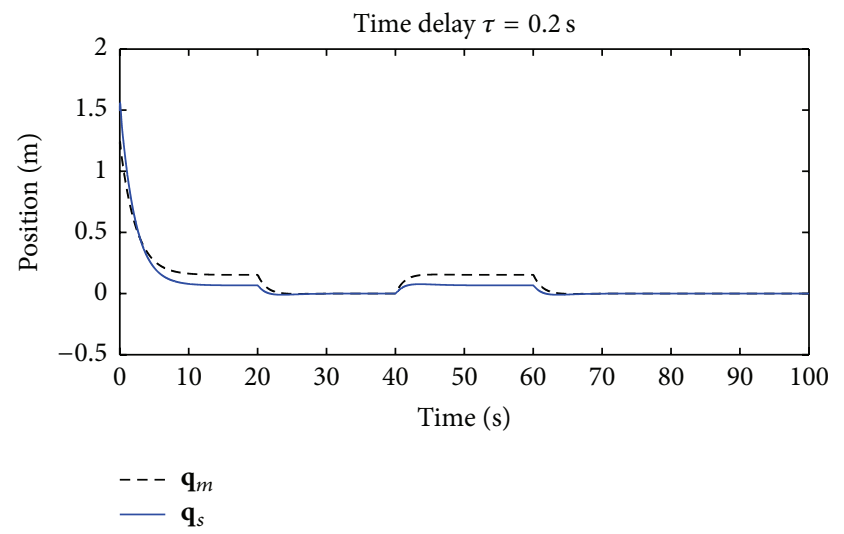

(a) Position tracking performance

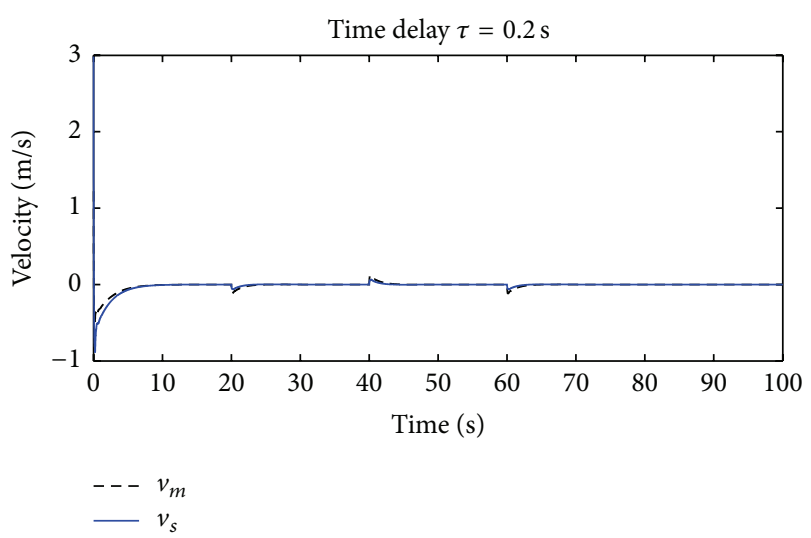

(b) Velocity tracking performance

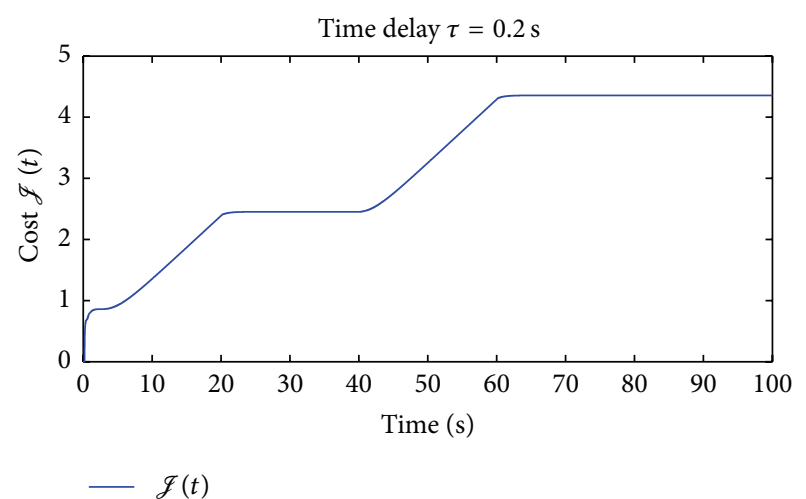

(c) Cost function $\mathscr{f}(t)$

FIgURE 5: Simulation results when human and environment forces are nonlinear.

bilateral teleoperation systems with complicated, nonlinear manipulator models, varying time delays, and parameter uncertainties.

\section{Conflict of Interests}

The authors declare that there is no conflict of interests regarding the publication of this paper. 


\section{Acknowledgment}

This work was supported by Key Program of National Natural Science Foundation of China (Grant no. 61333002).

\section{References}

[1] P. F. Hokayem and M. W. Spong, "Bilateral teleoperation: an historical survey," Automatica, vol. 42, no. 12, pp. 2035-2057, 2006.

[2] G. Niemeyer and J.-J. E. Slotine, "Telemanipulation with time delays," International Journal of Robotics Research, vol. 23, no. 9, pp. 873-890, 2004.

[3] R. J. Anderson and M. W. Spong, "Bilateral control of teleoperators with time delay," IEEE Transactions on Automatic Control, vol. 34, no. 5, pp. 494-501, 1989.

[4] G. Niemeyer and J.-J. E. Slotine, "Stable adaptive teleoperation," IEEE Journal of Oceanic Engineering, vol. 16, no. 1, pp. 152-162, 1991.

[5] E. Nuño, R. Ortega, N. Barabanov, and L. Basañez, "A globally stable pd controller for bilateral teleoperators," IEEE Transactions on Robotics, vol. 24, no. 3, pp. 753-758, 2008.

[6] E. Nũo, L. Basãez, R. Ortega, and M. W. Spong, "Position tracking for non-linear teleoperators with variable time delay," International Journal of Robotics Research, vol. 28, no. 7, pp. 895910, 2009.

[7] I. Sarras, E. Nuño, and L. Basañez, "An adaptive controller for nonlinear teleoperators with variable time-delays," Journal of the Franklin Institute, vol. 351, no. 10, pp. 4817-4837, 2014.

[8] S. Islam, P. X. Liu, and A. El Saddik, "Nonlinear adaptive control for teleoperation systems with symmetrical and unsymmetrical time-varying delay," International Journal of Systems Science, vol. 46, no. 16, pp. 2928-2938, 2015.

[9] N. Chopra, P. Berestesky, and M. W. Spong, "Bilateral teleoperation over unreliable communication networks," IEEE Transactions on Control Systems Technology, vol. 16, no. 2, pp. 304-313, 2008.

[10] S. Munir and W. J. Book, "Internet-based teleoperation using wave variables with prediction," IEEE/ASME Transactions on Mechatronics, vol. 7, no. 2, pp. 124-133, 2002.

[11] X. Yang, C. Hua, J. Yan, and X. Guan, "New stability criteria for networked teleoperation system," Information Sciences, vol. 233, pp. 244-254, 2013.

[12] Y. Q. Ye and P. X. Liu, "Improving trajectory tracking in wave-variable-based teleoperation," IEEE/ASME Transactions on Mechatronics, vol. 15, no. 2, pp. 321-326, 2010.

[13] Y.-C. Liu, “Task-space bilateral teleoperation systems for heterogeneous robots with time-varying delays," Robotica, 2014.

[14] H. L. Wang, "Task-space synchronization of networked robotic systems with uncertain kinematics and dynamics," IEEE Transactions on Automatic Control, vol. 58, no. 12, pp. 3169-3174, 2013.

[15] H. L. Wang and Y. C. Xie, "Passivity based task-space bilateral teleoperation with time delays," in Proceedings of the IEEE International Conference on Robotics and Automation (ICRA '11), pp. 2098-2103, Shanghai, China, May 2011.

[16] E. Nuño, R. Ortega, and L. Basañez, "An adaptive controller for nonlinear teleoperators," Automatica, vol. 46, no. 1, pp. 155-159, 2010.

[17] N. Chopra, M. W. Spong, and R. Lozano, "Synchronization of bilateral teleoperators with time delay," Automatica, vol. 44, no. 8, pp. 2142-2148, 2008.
[18] N. Chopra and M. W. Spong, "Adaptive coordination control of bilateral teleoperators with time delay," in Proceedings of the 43rd IEEE Conference on Decision and Control (CDC '04), vol. 5, pp. 4540-4547, Paradise Island, Bahamas, December 2004.

[19] E. Nuño, L. Basañez, and R. Ortega, "Passivity-based control for bilateral teleoperation: a tutorial," Automatica, vol. 47, no. 3, pp. 485-495, 2011.

[20] M. Kristalny and J. H. Cho, "On the decentralized $\mathrm{H}^{2}$ optimal control of bilateral teleoperation systems with time delays," in Proceedings of the 51st IEEE Annual Conference on Decision and Control (CDC '12), pp. 6908-6914, IEEE, Maui, Hawaii, USA, December 2012.

[21] L. Yu and J. Chu, "An LMI approach to guaranteed cost control of linear uncertain time-delay systems," Automatica, vol. 35, no. 6, pp. 1155-1159, 1999.

[22] H. K. Khalil, Nonlinear Systems, vol. 3, Prentice-Hall, Upper Saddle River, NJ, USA, 2nd edition, 2002.

[23] J. Löfberg, "YALMIP: a toolbox for modeling and optimization in MATLAB," in Proceedings of the IEEE International Symposium on Computer Aided Control System Design, pp. 284-289, September 2004. 


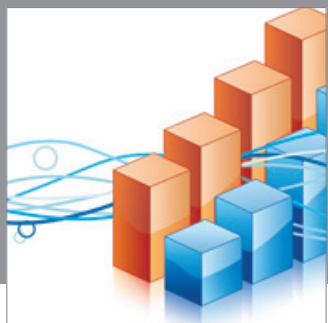

Advances in

Operations Research

mansans

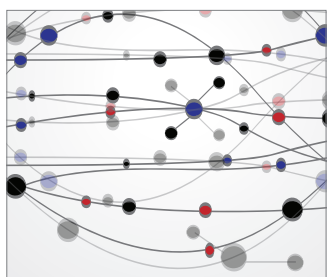

The Scientific World Journal
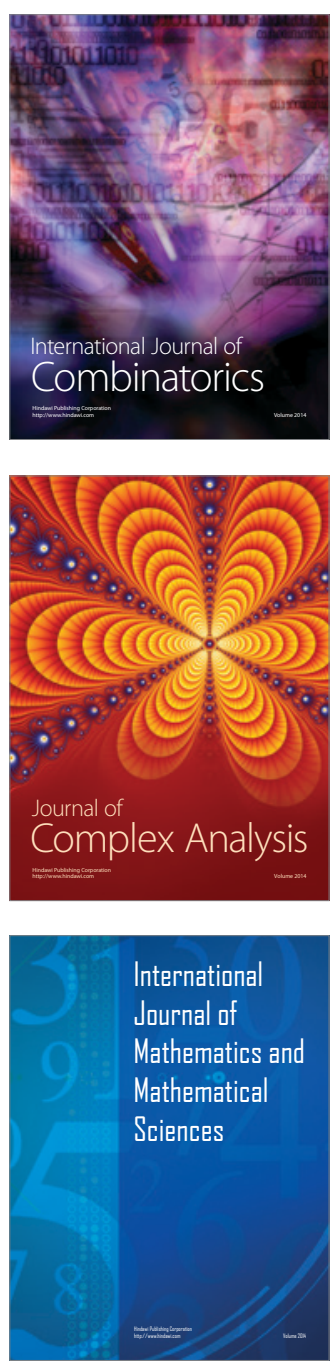
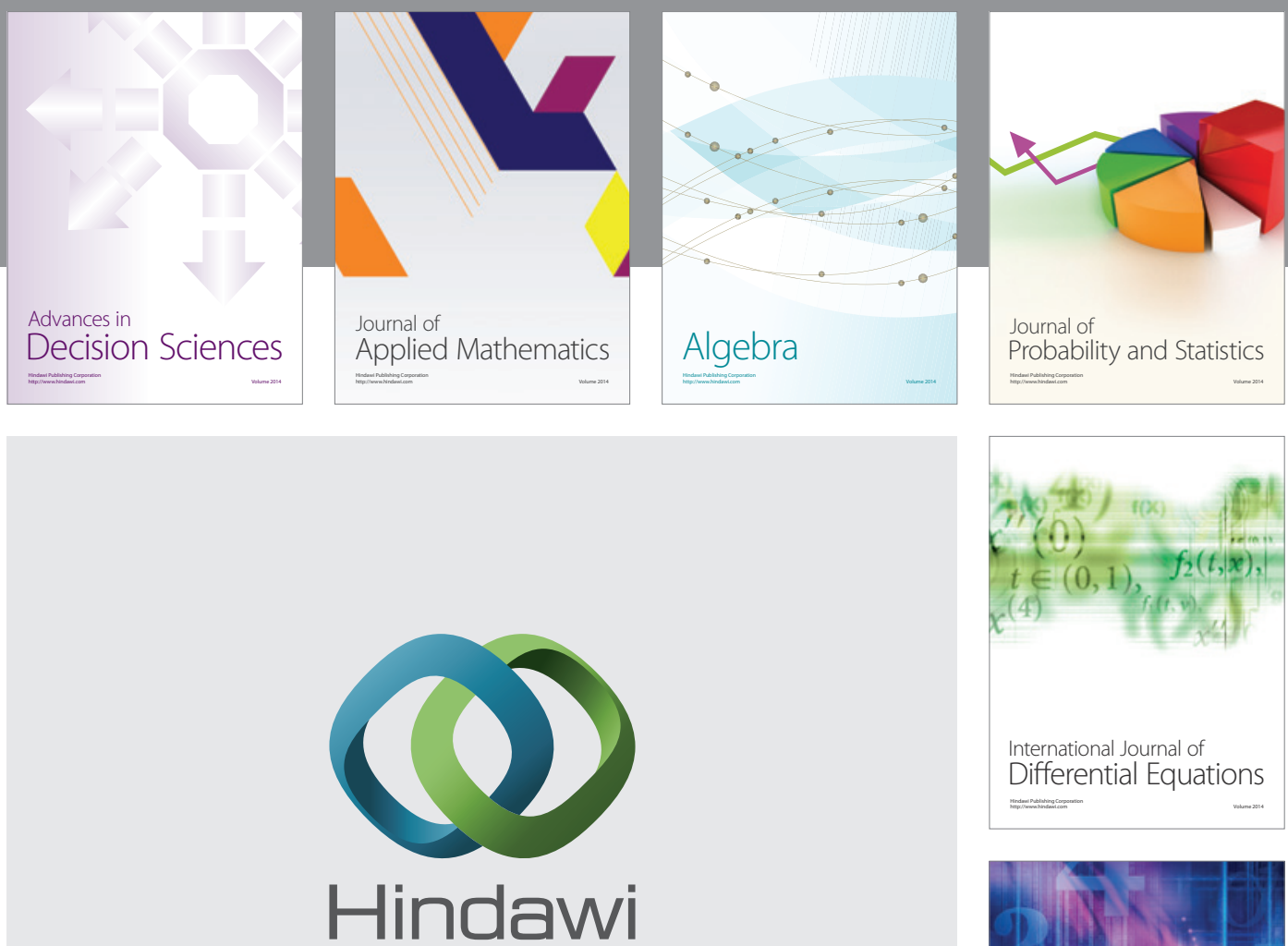

Submit your manuscripts at http://www.hindawi.com
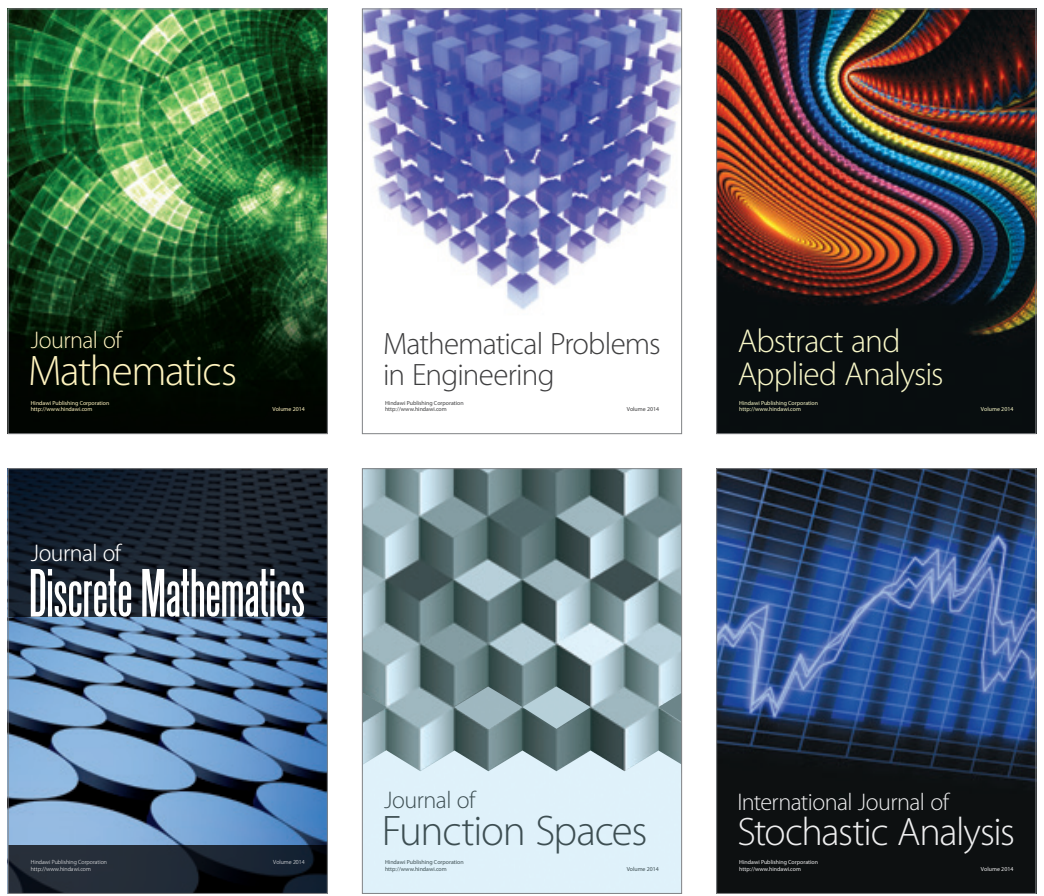

Journal of

Function Spaces

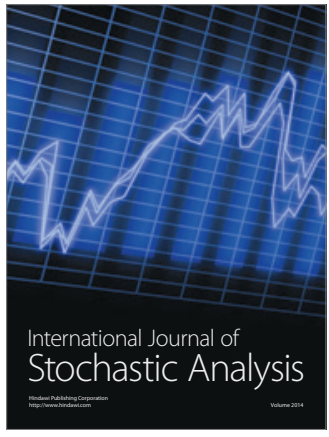

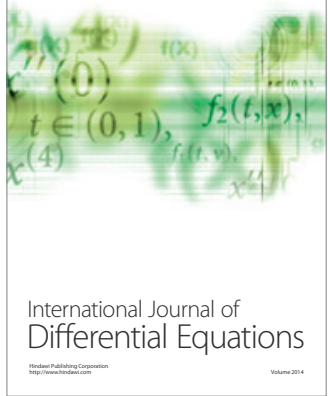
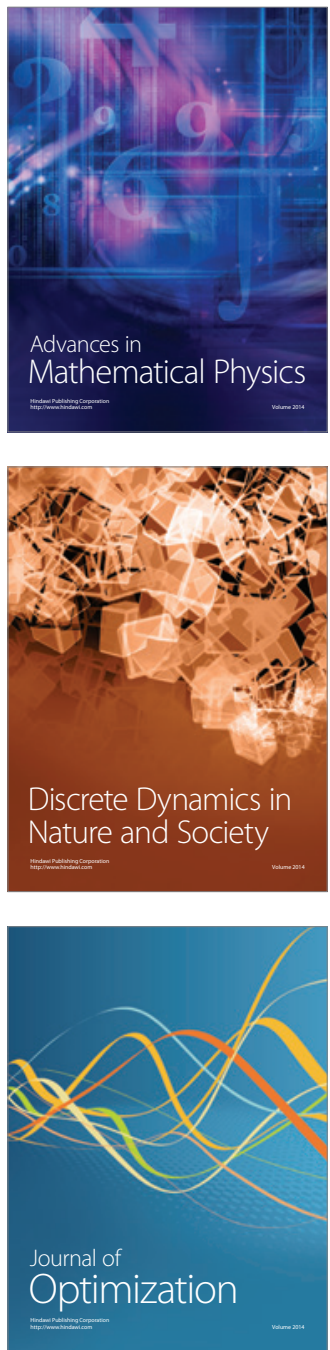